\title{
Comparison between Linear and Reverse Linear K-Space Order with Partial Fourier Fractions for Modulation Transfer Function in Dynamic Contrast-Enhanced Magnetic Resonance Imaging: A Simulation Study
} Takatsu $\mathrm{Y}^{1,2^{*}}$, Ueyama $\mathrm{T}^{3}$, Miyati $\mathrm{T}^{2}$ and Yamamura $\mathrm{K}^{2,4^{*}}$

${ }^{1}$ Department of Radiology, Osaka Red Cross Hospital, 5-30 Fudegasaki, Tennouji-ku, Osaka, 543-8555, Japan

${ }^{2}$ Division of Health Sciences, Graduate School of Medical Science, Kanazawa University, Japan

${ }^{3}$ Department of Radiology, The University of Tokyo Hospital, Japan

${ }^{4}$ Department of Radiology, Osaka Medical College Hospital, Japan

\begin{abstract}
Objective: To assess how the modulation transfer function (MTF) was influenced by the k-space trajectory with partial Fourier fractions in dynamic contrast-enhanced magnetic resonance imaging (DCE-MRI), calculating MTF using computer simulations.

Methods: Reference data for signal intensity were acquired using breast model at different concentrations and used to create a digital phantom. Frequency images were created by fast Fourier transform, divided into parts, and a new image formed by taking one part from each. The MTFs were then calculated. Three exponential curve models (slow, medium, and rapid) were created using the reference data.

Results: The smaller the partial Fourier fraction used, the faster the decline in MTF. MTF declined more rapidly with the linear order than with the reverse linear order.

Conclusion: The MTF was influenced by the k-space trajectory with partial Fourier fractions in DCE-MRI using computer simulations. The reverse linear order was found to be less influenced than the linear order by the partial Fourier fraction.
\end{abstract}

Keywords: Magnetic resonance imaging; Dynamic study; Partial fourier fraction; Modulation transfer function; K-space order; Digital phantom; Simulation

\section{Introduction}

The present study is part of a series of measurements of the modulation transfer function (MTF) that included in the previous study. Image contours are influenced by the partial Fourier fraction and the gradient of contrast medium concentration [1]. However, we assessed about the MTF using the only partial Fourier fraction. The MTF dependent on the k-space (frequency space) trajectory was not assessed.

Partial Fourier acquisition is used with dynamic contrast-enhanced magnetic resonance imaging (DCE-MRI) to reduce the scan time in the phase-encoding direction while preserving image resolution. The partial Fourier fraction is defined as the ratio of the partially acquired $\mathrm{k}$-space data size to the full $\mathrm{k}$-space data size; thus, acquiring half and full $\mathrm{k}$-spaces result in partial Fourier fractions of 0.5 and 1.0 , respectively. Typically, partial Fourier fractions in practice are between 0.55 and 0.75 [2]. The MTF can be used to assess image contours; however, several factors influence MTF measurement for MRI images. MTF measurements are degraded by a loss of system linearity, modulation of low spatial frequencies by non-uniformity effects, and underestimation of the measured value by several factors (such as chemical shift, T2 decay, ringing, and partial volume) [3-6]. To exclude these influences as far as possible, we created a digital phantom for simulated MTF measurements in our previous study, which investigated breast DCEMRI [1].

The concentration of the contrast medium changes over time; therefore, signal intensity changes, particularly during the early phase.
The signal intensity has been shown to increase to three times higher than the pre-administration intensity one minute after administration of the contrast medium $[7,8]$.

Various k-space trajectories can be used as methods of filling the $\mathrm{k}$-space (i.e., k-space ordering). Changing the k-space trajectory changes the filling timing of $\mathrm{k}-0$, the center of the $\mathrm{k}$-space. Thus far, however, there has been little investigation of how the image quality in dynamic studies varies according to the $\mathrm{k}$-space trajectory and different contrast medium concentrations, with the partial Fourier fractions calculated on digital phantoms. In the present study, therefore, we assessed MTF for evaluating image quality, using our previous simulation method for MTF measurement.

In general, data is filled from one edge to the other in the k-space, the so-called sequential order. We chose linear and reverse linear orders to compare different $\mathrm{k}$-space trajectories. In linear order, data are filled from left to right. When the partial Fourier fraction changes, the filling start position in the k-space changes, moving toward the center of the

*Corresponding author: Yasuo Takatsu, Department of Radiology, Osaka Red Cross Hospital, Japan, Tel: +81667745111; Fax: +81667745131; E-mail: pcblue2@yahoo.co.jp

Received May 12, 2017; Accepted May 23, 2017; Published July 01, 2017

Citation: Takatsu Y, Ueyama T, Miyati T, Yamamura K (2017) Comparison between Linear and Reverse Linear K-Space Order with Partial Fourier Fractions for Modulation Transfer Function in Dynamic Contrast-Enhanced Magnetic Resonance Imaging: A Simulation Study. J Comput Sci Syst Biol 10: 028-031. doi:10.4172/jcsb.1000245

Copyright: ( 2017 Takatsu Y, et al. This is an open-access article distributed unde the terms of the Creative Commons Attribution License, which permits unrestricted use, distribution, and reproduction in any medium, provided the original author and source are credited. 
Citation: Takatsu Y, Ueyama T, Miyati T, Yamamura K (2017) Comparison between Linear and Reverse Linear K-Space Order with Partial Fourier Fractions for Modulation Transfer Function in Dynamic Contrast-Enhanced Magnetic Resonance Imaging: A Simulation Study. J Comput Sci Syst Biol 10: 028-031. doi:10.4172/jcsb.1000245

$\mathrm{k}$-space (k-0) as the partial Fourier fraction is reduced. In reverse linear order, data are filled from right to left, the opposite direction of linear order. Use of the partial Fourier fraction does not change the filling start position for the reverse linear order (Figure 1). We therefore considered these k-space trajectories to be contrasting with each other and easy to compare. In our previous report, we used the same k-0 timing. Therefore, MTFs for different timings were not assessed. Accordingly, that study could not compare image quality with different timings of k-0 using MTF.

As the k-space can be characterized by Hermitian conjugate symmetry, blank areas can be compensated for (interpolation data) using corresponding portions of the filled data (collection data). We assumed that signal intensity differences between collection and interpolation data depended on the contrast medium concentration and on the partial Fourier fraction (Figure 2). Furthermore, we assumed that MTF was influenced by different filling start points in addition to those mentioned above. Therefore, we assessed how MTF was influenced by the k-space trajectory with partial Fourier fractions in DCE-MRI, calculating MTF using computer simulations.

\section{Materials and Methods}

\section{Digital phantom}

We used the same method as our previous study to create the digital phantom [1]. This method is briefly described below.

\section{Signal intensity of the concentration gradient model}

To assess the response of the signal intensity to changes in the contrast medium concentration, we created three exponential models (low, medium, and rapid) using reference data. In these models, the signal intensity varied from 500 to 1500 [1], evolving over time as follows:

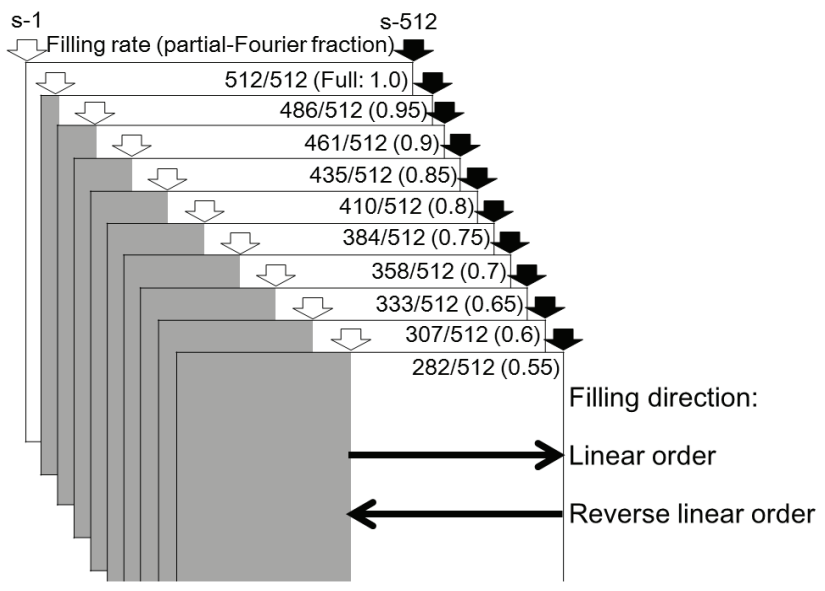

Figure 1: k-space order. To simulate for the calculation of MTF, k-space was made segmentation, from "s-1" to "s-512" (where "s" denotes "segment"). When the data fulfilling was complete, at linear order, the $512 \mathrm{k}$-spaces were ordered from left ("s-1" from the first k-space) to right ("s-512" from the 512th k-space). The beginning of the filling segment (white arrow) was variously. At reverse linear order, the $512 \mathrm{k}$-spaces were ordered opposite direction of linear order. The beginning of the filling segment (black arrow) was usually "s-512" from the first k-space. The filling rate (the partial Fourier fraction) was varied from $512 / 512$ to $282 / 512$ (i.e., from 1.0 to 0.55 ). When the actual data filled $486 / 512$ to $282 / 512$ of the k-space (partial Fourier fraction 0.95 0.55 ), the blank areas (gray areas) were filled with reflected data under point symmetry.
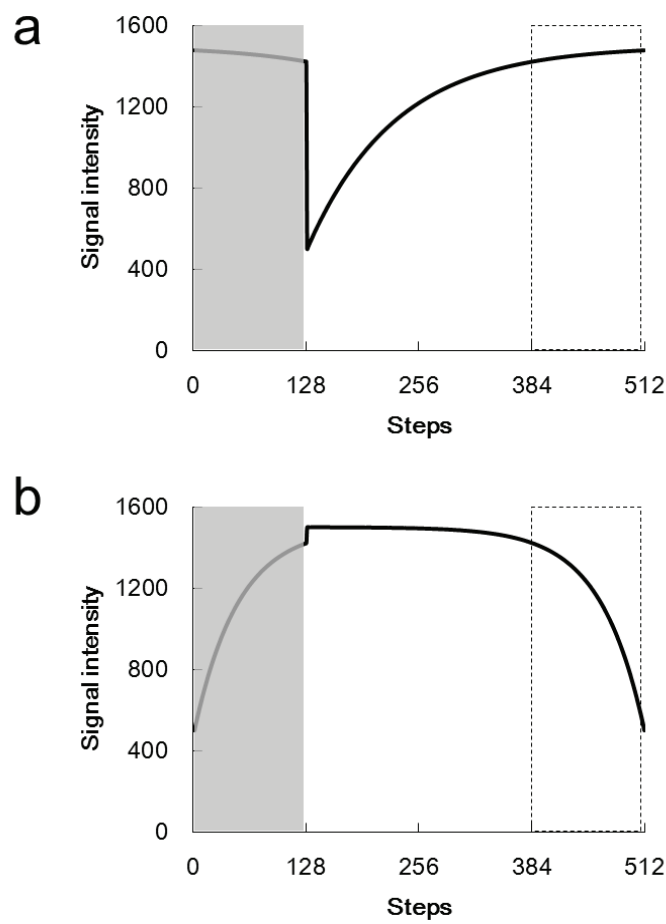

Figure 2: Difference of signal intensity between actual collection data and interpolation data (partial Fourier fraction, 0.75). The blank areas (gray areas) were filled with reflected data (dotted areas) under point symmetry; therefore, signal intensity difference between actual collection data and interpolation data was assumed to appear.

Signal intensity $=(A-C) \times[1-\exp (-k T)]+C$,

where $\mathrm{A}$ is the maximum signal intensity, $\mathrm{C}$ is the initial value, $\mathrm{T}$ is a time constant, and $\mathrm{k}$ is the coefficient of concentration gradient: slow $(\mathrm{k}=0.01)$, medium $(\mathrm{k}=0.02)$, or rapid $(\mathrm{k}=0.03)$. This exponential model was the same as the one used in a previous study. For details of this model, see reference 1 .

The imaging sequence parameter is arbitrary; therefore, concept of repetition time, echo time and so on was excluded.

\section{Imaging procedure}

The study followed a series of steps. (1) Digital phantoms were created. 512 images with $512 \times 512$ pixels were generated based on the time-dependent signal intensity in a square central area $(100 \times 100$ pixels) of the base signal area $(452 \times 452$ pixels). The signal intensity of the base signal area and initial value of square central area were 500 . (2) The digital phantoms were transformed by fast Fourier transform (FFT). (3) Each resultant image was divided into 512 strips (Figures 3 and 4). (4) One strip from each FFT image was taken and arranged into a composite image of linear and reverse linear orders (Figures 3 and 4). The blank strips were filled with reflections of data from the early k-spaces. (5) The inverse FFT of the composite image was computed (Figures 3 and 4). (6) MTF was calculated from the composite image as described below. The digital phantom was created in Excel 2013 (Microsoft Corp., Redmond, WA, USA), and all image calculations were performed using ImageJ software (National Institutes of Health, Bethesda, MD, USA). 
Citation: Takatsu Y, Ueyama T, Miyati T, Yamamura K (2017) Comparison between Linear and Reverse Linear K-Space Order with Partial Fourier Fractions for Modulation Transfer Function in Dynamic Contrast-Enhanced Magnetic Resonance Imaging: A Simulation Study. J Comput Sci Syst Biol 10: 028-031. doi:10.4172/jcsb.1000245

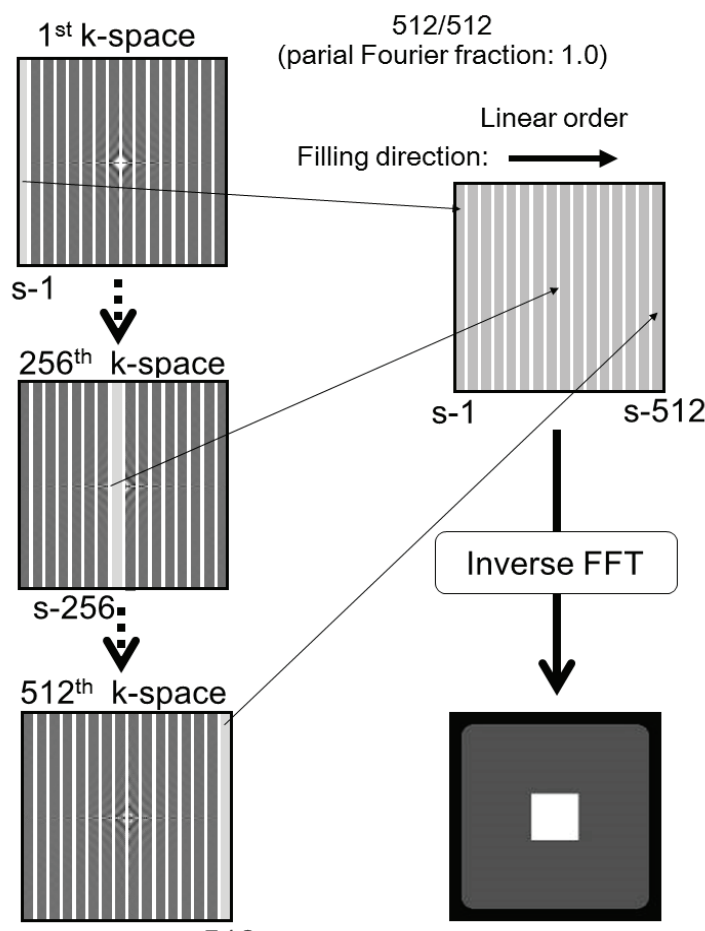

s-512

Figure 3: k-space filling of the linear order. The $512 \mathrm{k}$-spaces were divided into 512 parts ("s-1" to "s-512" from left to right, each of $512 \times 1$ pixels). One part was extracted from each k-space. The $512 \mathrm{k}$-spaces were ordered from left ("s-1" from the first k-space) to right ("s-512" from the 512th k-space) when the data fulfilling was complete. The beginning of the filling segment was variously (Figure 1). The extracted parts were arranged in order and subjected to inverse FFT, yielding time-dependent images from the real and imaginary data.

\section{Calculation of MTF}

MTF was calculated by a three-step procedure. First, an edge spread function (ESF) was created. Second, this ESF was differentiated to obtain the line spread function (LSF). Third, MTF was obtained by calculating FFT of LSF. The resultant values of MTF were compared for different partial Fourier fractions and k-space trajectories.

\section{Results}

MTF declined more rapidly with smaller partial Fourier fractions (Figure 5). The most stable MTF was that for the rapid exponential model. MTF declined more rapidly with the linear order than with the reverse linear order. With smaller partial Fourier fractions in the linear order, MTF increased to more than 1 . This effect became stronger as the coefficient of concentration gradient was reduced.

\section{Discussion}

In the linear order, MTF increased to more than 1 . The difference in signal intensity between the collection and interpolation of data by the gradient of the contrast medium concentration was large; therefore, the image contour deteriorated, leading to overshoot and undershoot, and then the shape of the LSF was influenced. The information of the image contour, including the invasion of the tumor, was important element for diagnose.

In the linear order, the filling start position was closer to the $\mathrm{k}-0$ for smaller partial Fourier fractions; i.e., it became more similar to the centric order. When the partial Fourier fraction was larger, the filling start position was closer to the sequential order, i.e., closer to the reverse linear order.

Data of k-0 contributed to the image contrast. MTF may have been influenced by the larger difference in signal intensity between collection and interpolation data. In the reverse linear order, the filling time of $\mathrm{k}-0$ came at the midpoint of the full filling. This point never changed. However, in the linear order, the filling time of k-0 changed depending on the partial Fourier fraction.

To increase the tissue contrast using the contrast medium, the timing of k-0 filling was important and was influenced by the injection rate. Thus, an adjustment should be needed; however, the reverse linear order was less influenced by the partial Fourier fraction. Furthermore, if the timing for the contrast medium reaching $\mathrm{k}-0$ was adjusted, high tissue contrast could be obtained. However, contrast enhancement magnitude and kinetics would depend on the tissue type.

Among the exponential models, the rapid exponential model yielded the highest MTF. This was because its long plateau ensured the longest stable intensity term. This result corresponded to the results of our previous report [1].

This study had several limitations. We simulated only the filling of the contrast medium in early phase, and not the wash-out. The rationale for this was that we considered that there would be a greater influence on MTF during fill-in than during wash-out because of the

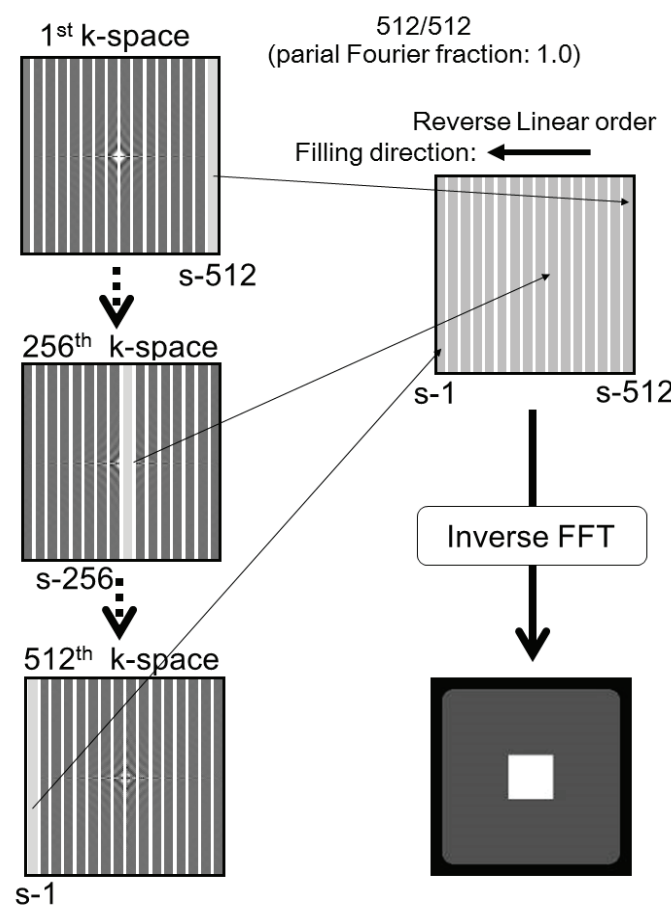

Figure 4: k-space filling of the reverse linear order. The $512 \mathrm{k}$-spaces were divided into 512 parts same as Figure 3 . One part was extracted from each k-space. The $512 \mathrm{k}$-spaces were ordered opposite direction of linear order. The data was filled from right ("s-512" from the first k-space) to left ("s-1" from the 512th k-space) when the data fulfilling was complete. The beginning of the filling segment was usually "s-512" from the first k-space. The extracted parts were arranged in order and subjected to inverse FFT, yielding timedependent images from the real and imaginary data. 
Citation: Takatsu Y, Ueyama T, Miyati T, Yamamura K (2017) Comparison between Linear and Reverse Linear K-Space Order with Partial Fourier Fractions for Modulation Transfer Function in Dynamic Contrast-Enhanced Magnetic Resonance Imaging: A Simulation Study. J Comput Sci Syst Biol 10: 028-031. doi:10.4172/jcsb.1000245

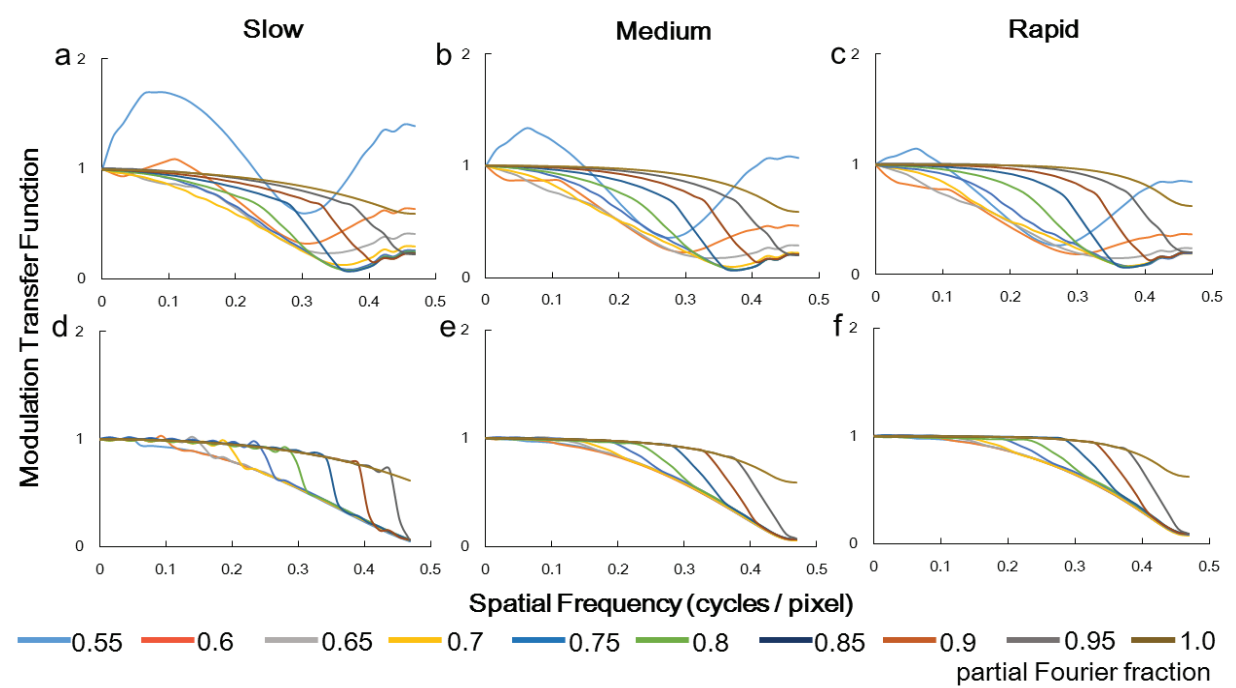

Figure 5: Result of calculation for the MTF. The MTF of linear order for three exponential models; slow (a), medium (b), and rapid (c), and reverse linear order for three exponential models; slow (d), medium (e), and rapid (f) were shown. The most stable MTF was that for the rapid exponential model. MTF declined more rapidly with the linear order than with the reverse linear order.

rapid change in contrast medium concentration. In this study, we ignored the effects of the echo time on the signal strength. The signal strength could also be influenced by the k-space trajectory. However, we excluded such a factor to obtain pure data regarding the influence of the k-space trajectory with the partial Fourier fraction in DCE-MRI as a simulation. In this study, the injection timing was stable; however, if the injection timing were to change, this could influence the shape of MTF.

To make a contrast, centric order is easy to use for the timing of contrast enhancement, especially arterial input function [9]; however, we did not evaluate the centric order.

The image quality assessment that several algorithms were used for partial Fourier acquisition was reported [10]. However, conventional conjugate symmetry was used for this study.

To increase study speed, the partial Fourier method has been combined with parallel imaging acquisition [11-13]. However, the aim of our study was to investigate the influence of altering the gradient of the contrast medium with the k-space trajectory on MTF. By doing this, we obtained the important result that the reverse linear order was less influenced by the partial Fourier fraction.

\section{Conclusion}

The MTF was influenced by the k-space trajectory with partial Fourier fractions in DCE-MRI using computer simulations. The reverse linear order was found to be less influenced than the linear order by the partial Fourier fraction.

\section{Acknowledgements}

Nothing in particular.

\section{Disclosure of Conflicts of Interest}

The authors declare that they have no conflict of interest. This article does not contain any studies with human participants performed by any of the authors.

\section{References}

1. Takatsu Y, Ueyama T, Miyati T, Kenichirou Y (2016) Simulation of the modulation transfer function dependent on the partial Fourier fraction in dynamic contrast enhancement magnetic resonance imaging. Australas Phys Eng Sci Med 39: 825-831.

2. Bernstein MA, King KF, Zhou XJ (2004) Handbook of MRI Pulse Sequences Partial Fourier Reconstruction. ElsevierAcademic Press, London, UK, pp: 546-558.

3. Steckner MC, Drost DJ, Prato FS (1991) Comments and Reply: "Transfer function measurements and analysis for a magnetic resonance imager". Mohapatra SM [Med Phys. 18: 1141-1144.]. Med Phys 19: 511-512.

4. Steckner MC, Drost DJ, Prato FS (1993) A cosine modulation artifact in modulation transfer function computations caused by the misregistration of line spread profiles. Med Phys 20: 469-473.

5. Steckner MC, Drost DJ, Prato FS (1994) Computing the modulation transfer function of a magnetic resonance imager. Med Phys 21: 483-489.

6. Miyati T, Fujita H, Kasuga T, Sanada S, Fujita H, et al. (2001) MTF measurement in MRI using a complex subtraction method. J Radiol Technol 57: 1225-1232.

7. Kelcz F, Furman-Haran E, Grobgeld D, Degani H (2002) Clinical testing of highspatial-resolution parametric contrast-enhanced MR imaging of the breast. AJR 179: $1485-1492$

8. Takeda Y, Yoshikawa K (2002) Contrast-enhanced dynamic MR imaging parameters and histological types of invasive ductal carcinoma of breast. Biomed Pharmacother 59: 115-121.

9. Kim D (2008) Influence of the k-space trajectory on the dynamic T1-weighted signal in quantitative first-pass cardiac perfusion MRI at 3T. Magn Reson Med 59: 202-208.

10. McGibney G, Smith MR, Nichols ST, Crawley A (1993) Quantitative evaluation of several partial Fourier reconstruction algorithms used in MRI. Magn Reson Med 30: 51-59.

11. Bydder M, Robson MD (2005) Partial Fourier partially parallel imaging. Magn Reson Med 53: 1393-1401.

12. King KF, Angelos L (2000) SENSE with partial Fourier homodyne reconstruction Proc Intl Sot Magn Reson Med 8: 153.

13. Ponce IP, Blaimer M, Breuer FA, Griswold MA, Jakob PM, et al. (2014) Autocalibration approach for k-t SENSE. Magn Reson Med 71: 1123-1129. 